\title{
Relationship of side dominance and ultrasonographic measurements of pronator quadratus muscle along with handgrip and pinch strength.
}

\author{
Nusret Ok ${ }^{1}$, Kadir Agladioglu² ${ }^{2}$ Harun R. Gungor ${ }^{1}$, Ali Kitis ${ }^{3}$, Semih Akkaya ${ }^{1}$, Nuran Sabir \\ Akkoyunlu ${ }^{2}$, Fahir Demirkan ${ }^{1}$
}

${ }^{1}$ Pamukkale University Medical Faculty Orthopedics and Traumatology Department, ${ }^{2}$ Pamukkale University Medical Faculty Radiology Department, ${ }^{3}$ Pamukkale University School of Physical Therapy and Rehabilitation Department, Denizli, Turkey

\begin{abstract}
Aims: We ascertained effects of hand dominance in both right and left-handed volunteers on the thickness and cross sectional area (CSA) of pronator quadratus (PQ) muscle as measured by the axial and sagittal views of ultrasound imaging. Secondly, we also aimed to document the extent of the relationship between the PQ measurements and handgrip. Materials and methods: Healthy adults from the volunteer medical staff were included in the study. Handedness was assessed via The Flinders Handedness survey. The sonographic appearance of the PQ was evaluated on sagittal and axial images for both hands. CSA of PQ muscle was digitally drawn and calculated on the axial plane. Handgrip was measured by using adjustable-handle dynamometer. Results: Eighty-nine healthy volunteers were included (54 right and 35 left-handed). Significant difference was detected between dominant and non-dominant hands in CSA and muscle thickness in both right and left-handed volunteers $(\mathrm{p}<0.05)$. These measurements were correlated with the handgrip strength $(\mathrm{p}<0.05, \mathrm{r}=0.55$, and $\mathrm{r}=0.43$ for right-handed volunteers, $r=0.67$ and $r=0.48$ for left-handed volunteers, respectively). There were also significantly high correlations between the measurements of CSA and PQ thickness of the corresponding extremity in both right-handed and left-handed volunteers $(p<0.05)$. Conclusions: A statistically significant difference exists between dominant and non-dominant hands in CSA and muscle thickness measured by US in both right and left handed volunteers, and this is correlated with handgrip strength. Hand dominance should be considered to compare healthy and affected sites during US of PQ while investigating for occult fractures of distal forearm or PQ atrophy due to anterior interosseous nerve injury.
\end{abstract}

Keywords: handedness, pronator quadratus muscle, ultrasonography, cross sectional area, handgrip

\section{Introduction}

The pronator quadratus (PQ) muscle inserts distally $1 / 6^{\text {th }}$ of the anterior surface of radio-ulnar joint. While the superficial layer of PQ acts in coordination with the pronator teres and serves for forearm pronation, deep layer

Received 15.11.2015 Accepted 15.02.2016

Med Ultrason

2016, Vol. 18, No 2, 170-176

Corresponding author: Harun R. Gungor, MD

Pamukkale University Medical Faculty Orthopedics and Traumatology Department 20070, Kinikli / Denizli / Turkey

Phone: +90 2582965670

Mobile: +905322343770

Fax: +902582966001

E-mail: hrgungor@gmail.com inserts joint capsule and take part in radio-ulnar joint stabilization [1-3]. In addition, both heads of the pronator quadratus also play a role in grip strength [4]. The obliteration of the fat plane between the pronator quadratus and the flexor digitorum profundus muscles which is a radiolucent plane that can be detected on lateral radiographs of the distal forearm namely the pronator sign has been reported since 1960s as an important diagnostic tool for the detection of the occult distal radius and ulna traumatic fractures [5]. However, sensitivity and specificity of this sign on plain radiographs for diagnosis of distal forearm fractures are quite low in contrast to magnetic resonance imaging (MRI) [6].

Although MRI is a reliable method in the diagnosis of occult bone injury this examination has some drawbacks since it is a time consuming and costly procedure. There- 
fore, PQ ultrasonography (US) has been investigated in several studies for the detection of occult distal forearm bone injuries or anterior interosseous nerve injuries offering advantages in cost and comparison with healthy sites [7-10]. Sato et al [8] reported that sonographic swelling of PQ in contrast to the healthy site in patients without findings in lateral radiographs of the distal forearm following acute injury might be an indicator for occult bone injury. However, the difference in the pronator quadratus thickness between dominant and non-dominant hands and isolated PQ atrophy without anterior intraosseous nerve entrapment in asymptomatic healthy individuals may be a limiting factor during sonographic studies of PQ in patients with an acute occult bone injury in distal forearm $[7,9,10]$. Although, effect of hand dominance on PQ thickness has been investigated in healthy right handed volunteers, there has been no studies covering both right and left handed individuals comparing PQ thickness and cross sectional area (CSA) between dominant and non-dominant hands.

Here, we ascertain effects of hand dominance in both right and left handed individuals on the thickness of the PQ muscle and CSA as measured by axial and sagittal views of ultrasound imaging. Secondarily, we also aimed to document the extent of the relationship between the PQ thickness and handgrip concerning PQ's stabilizing effect on distal radio-ulnar joint in addition to its pronator function.

\section{Materials and methods}

Following approval of the Ethical Committee (Pamukkale University Medical Faculty, Denizli, Turkey; No: 60116787-020/61419), healthy adults from the volunteer medical staff were included in the study. Volunteers with a history of major upper extremity trauma, fracture, chronic systemic problems related to musculoskeletal system, recent hospitalization, and surgery were excluded from the study.

To determine if subjects met the above criteria, a brief interview was conducted which preceded all testing. Following the interview, hand preferences of the volunteers were detected by applying a survey for handedness. An independent observer other than the observers performing ultrasonography and hand strength measurements, performed this step and referred the volunteers to the blinded observers for sonographic measurements of PQ and strength assessment of both hands.

\section{Assessment of Handedness}

Handedness was assessed via The Flinders Handedness survey (FLANDERS); a measure of skilled hand preference originally reported by Nicholls et al [11]. Re- sponses of 10 questions being 'left', 'either' and 'right' are assigned scores of $-1,0$ and +1 , respectively. These scores are then totalled to give a test score that ranges from -10 to +10 . Individuals with scores ranging between -10 and -5 were designated to be left-handed whereas individuals with scores ranging between +5 and +10 were designated as right-handed. Individuals with scores between these ranges were considered to be mixed-handed or ambidextrous.

\section{Ultrasound Measurements}

The volunteers sitting on a chair placed their hands on a rectangular table. Hands were positioned fully relaxed during the whole ultrasonographic evaluation with the shoulder adducted and neutrally rotated, elbow flexed at $90^{\circ}$, forearm in neutral position, and wrist between $5^{\circ}$ and $10^{\circ}$ dorsiflexion. The sonographic appearance of the PQ was evaluated on sagittal and axial images for both hands. The sagittal image was used to measure the thickness of the radial part of the PQ (represents mainly radial part of superficial layer) and the axial image was used to measure mainly the thickness of the interosseous and ulnar parts of superficial layer [7,12]. For the sagittal image, the probe was positioned along the flexor carpi radialis tendon on the volar aspect of the wrist, and the maximum thickness of the PQ was measured at corresponding sites of both hands [7,12] (fig 1a). The flexor carpi radialis, $\mathrm{PQ}$, volar cortex of the radius, and the bony prominence attached to the PQ were included in the sagittal image. Since the sagittal image mainly represents the superficial layer of PQ on the volar side at the radial cortical level, we considered the correspondence to the radiographic PQ sign and measured the maximum thickness at this level $[7,12]$. For the axial image of the PQ muscle, the probe was positioned distally adjacent to the radiocarpal joint and moved proximally from carpus to forearm to get the maximum thickness on the volar side at the cortical level. For this measurement, a tangential line beginning from the ulnar rim of the radius to the convex site of the ulna was drawn, and maximum thickness of the muscle was measured perpendicular to this line [7,12] (fig 1b). Cross sectional boundaries of the PQ muscle area on this axial plane where the maximum thickness of the muscle measured was digitally drawn to evaluate both superficial and deep parts, and the software of US machine automatically calculated CSA (Fig 1c). Three successive measurements were performed on the same occasion and average results were recorded. The examinations were performed using a sonographic scanner (Logiq E9, GE Medical Systems, Wauwatosa, WI, USA) and 11-15 MHz linear prob. To assess intra-observer reliability, the main observer of US measurements (KA) performed two separate measurements on the first 


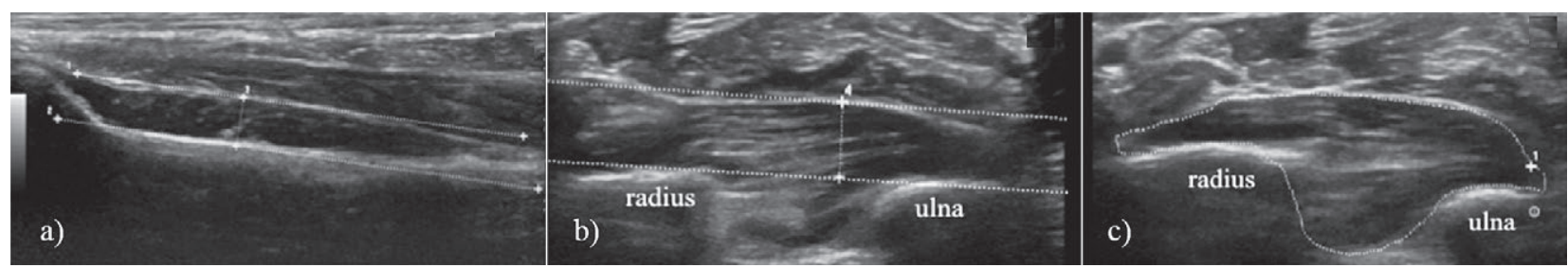

Fig 1. Measurements of pronator quadratus: in the sagittal view (a), in the axial view (b), drawing of cross sectional area to include both superficial and deep layers in the axial view (c).

30 volunteers with one-week interval. To assess interobserver reliability an additional observer (HRG) also performed US measurements on the first 30 volunteers.

\section{Assessment of Hand Strength}

The volunteers were seated with their shoulder adducted and neutrally rotated, elbow flexed at $90^{\circ}$, forearm in neutral position, and wrist at $0^{\circ}$ to $30^{\circ}$ dorsiflexion, and $0^{\circ}$ to $15^{\circ}$ ulnar deviation $[13,14]$. Handgrip strength was tested first (fig 2a), followed by nail-to-nail pinch (pinch 1), tip (two-point) pinch (pinch 2), key (lateral) pinch (pinch 3), and palmar (three-jaw chuck) pinch (pinch 4) (fig 2b). Standardized instructions described by Mathiowetz et al [15] were used for testing. The standard, adjustable-handle Jamar dynamometer, reported as the most accurate for measuring grip strength, was used in the measurements [16]. For standardization, it was set at the second handle position for all subjects $[16,17]$. The dynamometer was lightly held around the readout dial by the examiner to prevent inadvertent dropping. Scores were read on the needle side of the red readout marker. One observer (AK) performed all of the measurements, and the scores of three successive trials were recorded for both hands for each strength test. The calibration of instruments was tested periodically during the examinations $[16,17]$.

\section{Statistical Analysis}

An a priori power analysis (tested against a constant of 0.00 ) proved that at least 34 volunteers were required to detect a significant difference between the PQ thickness of dominant and non-dominant hands in left handed individuals with $90 \%$ power $(\mathrm{p}<0.05 ; \alpha=0.05 ; 2$ tailed; confidence interval $95 \%$ ). An a posteriori power analysis (tested against a constant of 0.00 ) showed $95 \%$ power to detect a significant difference between PQ thickness of dominant and non-dominant hands in left handed individuals with 35 people $(\mathrm{p}<0.05 ; \alpha=0.05 ; 2$ tailed; confidence interval 95\%). The data were analyzed with Statistical Package for Social Sciences software (SPSS Version 18, Chicago, IL, USA). Descriptive statistics (including mean \pm standard deviation, frequency, and percentage) were calculated for both right and left-handed volunteers. For parametric tests, the difference between the means of

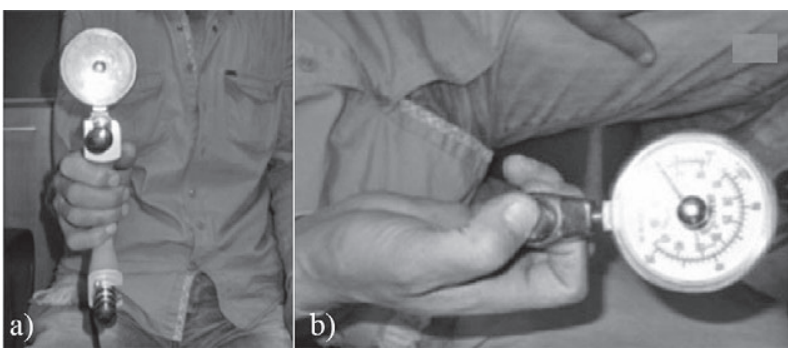

Fig 2. Handgrip (a) and pinch strength (b) measurements using the dynamometer.

variables in groups was compared using the independent samples t test. Pearson's correlation coefficient was used to determine a correlation between the muscle strength and US measurements. Statistical significance was set at $\mathrm{p}<0.05$. Interclass correlation coefficient (confidence interval 95\%) was used for intra- and inter-observer reliability.

\section{Results}

Eighty-nine healthy volunteers were included in the study (54 right handed and 35 left handed; $49 \mathrm{M}$ and 40 F; age range between 20-50 years; mean age 28,74 $\pm 6,98$ years). Demographic and physical characteristics of patients are summarized on Table I.

Intra-observer reliability was high for sagittal and axial thickness measurements for both right and left hands (ICC values for axial thickness of right hand $=0.980$, of left hand $=0.985, \mathrm{p}=0.001$ with $95 \% \mathrm{CI}$; ICC values for sagittal thickness of right hand $=0.972$, of left hand $=0.974$, $\mathrm{p}=0.001$ with $95 \% \mathrm{CI}$ ). Inter-observer reliability was high for axial and sagittal thickness measurements both right and left hands (ICC values for axial thickness of right hand $=0.993$, of left hand $=0.991, p=0.001$ with $95 \%$ CI; ICC values for sagittal thickness of right hand $=0.994$, of left hand $=0.990, \mathrm{p}=0.001$ with $95 \% \mathrm{CI}$ ).

No volunteer was classified as ambidextrous in our study. US measurements of PQ, grip strength, and pinch strength results in right-handed and in left-handed volunteers are summarized on Table II and III, respectively. 
Table I. Demographic and physical characteristics of the volunteers

\begin{tabular}{lllllll}
\hline Volunteers & $\begin{array}{l}\text { Age } \\
\text { mean } \pm \text { SD (min-max) }\end{array}$ & $\begin{array}{l}\text { Sex } \\
(\mathbf{M}, \mathbf{F})\end{array}$ & $\begin{array}{l}\text { Weight } \\
\text { mean } \pm \text { SD (min-max) }\end{array}$ & $\begin{array}{l}\text { Heigth } \\
\text { mean } \pm \text { SD (min-max })\end{array}$ & $\begin{array}{l}\text { BMI* } \\
\text { mean } \pm \text { SD (min-max) }\end{array}$ & p \\
\hline Right-handed $(\mathrm{n}=54)$ & $28.4 \pm 6.4(21-45)$ & $(30,24)$ & $70.4 \pm 10.5(45-90)$ & $175 \pm 11(150-190)$ & $22.9 \pm 1.5(20-25)$ & $\mathrm{n} . \mathrm{s} . \dagger$ \\
Left-handed $(\mathrm{n}=35)$ & $29.3 \pm 7.9(20-50)$ & $(19,16)$ & $71.1 \pm 12.2(49-94)$ & $172 \pm 11(150-192)$ & $23.8 \pm 2.1(20-28)$ & $\mathrm{n} . \mathrm{s} . \dagger$ \\
Total $(\mathrm{n}=89)$ & $28.7 \pm 6.9(20-50)$ & $(49,40)$ & $70.7 \pm 11.1(45-94)$ & $174 \pm 11(150-192)$ & $23.2 \pm 1.8(20-28)$ & n.s. $\dagger$ \\
\hline
\end{tabular}

*Body mass index; $\uparrow$ Not significant $(\mathrm{p}>0.05)$

Table II. Results of grip strength, pinch strength and ultrasonography measurements in right-handers.

\begin{tabular}{|c|c|c|c|c|c|}
\hline & & $\mathbf{n}$ & Mean \pm SD & $\mathbf{t}$ & p value \\
\hline Grip (kg) & $\begin{array}{l}\mathrm{R} \\
\mathrm{L}\end{array}$ & 54 & $\begin{array}{l}33.14 \pm 11.34 \\
31.62 \pm 12.23\end{array}$ & 3.25 & $<0.05$ \\
\hline Pinch-1 (kg) & $\begin{array}{l}\mathrm{R} \\
\mathrm{L}\end{array}$ & 54 & $\begin{array}{l}3.99 \pm 1.99 \\
3.36 \pm 1.73\end{array}$ & 4.73 & $<0.05$ \\
\hline Pinch-2 (kg) & $\begin{array}{l}\mathrm{R} \\
\mathrm{L}\end{array}$ & 54 & $\begin{array}{l}4.75 \pm 1.82 \\
4.27 \pm 1.69\end{array}$ & 3.82 & $<0.05$ \\
\hline Pinch-3 (kg) & $\begin{array}{l}\mathrm{R} \\
\mathrm{L}\end{array}$ & 54 & $\begin{array}{l}6.14 \pm 2.04 \\
5.68 \pm 2.05\end{array}$ & 3.76 & $<0.05$ \\
\hline Pinch-4 (kg) & $\begin{array}{l}\mathrm{R} \\
\mathrm{L}\end{array}$ & 54 & $\begin{array}{l}3.62 \pm 1.32 \\
3.69 \pm 1.99\end{array}$ & -0.38 & n.s. \\
\hline Axial (mm) & $\begin{array}{l}\mathrm{R} \\
\mathrm{L}\end{array}$ & 54 & $\begin{array}{l}6.4 \pm 1.3 \\
5.6 \pm 1.3\end{array}$ & 10.34 & $<0.05$ \\
\hline Sagital (mm) & $\begin{array}{l}\mathrm{R} \\
\mathrm{L}\end{array}$ & 54 & $\begin{array}{l}4.2 \pm 1 \\
3.8 \pm 0.9\end{array}$ & 8.53 & $<0.05$ \\
\hline $\mathrm{CSA}\left(\mathrm{mm}^{2}\right)$ & $\begin{array}{l}\mathrm{R} \\
\mathrm{L}\end{array}$ & 54 & $\begin{array}{l}21.9 \pm 5.6 \\
19.7 \pm 4.6\end{array}$ & 8.14 & $<0.05$ \\
\hline
\end{tabular}

$\mathrm{n}$ - number of volunteers, CSA - Cross sectional area, $\mathrm{R}$ - right, $\mathrm{L}-$ left, n.s - not significant

Table IV. Correlation between the grip strength, pinch strength and ultrasonography measurement results in right-handed volunteers $(n=54)$.

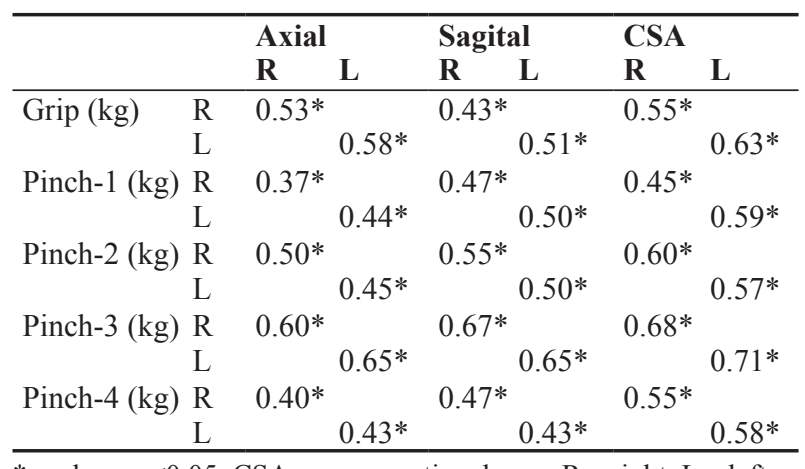

${ }^{*} \mathrm{r}$ values, $\mathrm{p}<0.05, \mathrm{CSA}-$ cross sectional area, $\mathrm{R}$ - right, $\mathrm{L}-$ left

For right-handers, all the measurements were found to be significantly higher $(\mathrm{p}<0.05)$ on the dominant side except the three-jaw chuck pinch ( $p>0.05)$. For left-handers, all the measured parameters were found to be slightly higher in the left hand rather than the right hand except in the case of the three-jaw chuck pinch $(p<0.05)$. However,
Table III. Results of the grip strength, pinch strength and ultrasonography measurements in left-handers

\begin{tabular}{|c|c|c|c|c|c|}
\hline & & $\mathbf{n}$ & Mean \pm SD & $\mathbf{t}$ & p value \\
\hline Grip (kg) & $\begin{array}{l}\mathrm{R} \\
\mathrm{L}\end{array}$ & 35 & $\begin{array}{l}34.16 \pm 11.79 \\
34.76 \pm 11.03\end{array}$ & -0.86 & n.s \\
\hline Pinch-1 (kg) & $\begin{array}{l}\mathrm{R} \\
\mathrm{L}\end{array}$ & 35 & $\begin{array}{l}4.48 \pm 2.02 \\
4.49 \pm 1.90\end{array}$ & -0.05 & n.s. \\
\hline Pinch-2 (kg) & $\begin{array}{l}\mathrm{R} \\
\mathrm{L}\end{array}$ & 35 & $\begin{array}{l}4.98 \pm 1.93 \\
5.08 \pm 1.80\end{array}$ & -0.49 & n.s. \\
\hline Pinch-3 (kg) & $\begin{array}{l}\mathrm{R} \\
\mathrm{L}\end{array}$ & 35 & $\begin{array}{l}6.30 \pm 2.42 \\
6.45 \pm 2.03\end{array}$ & -0.84 & n.s. \\
\hline Pinch-4 (kg) & $\begin{array}{l}\mathrm{R} \\
\mathrm{L}\end{array}$ & 35 & $\begin{array}{l}4.11 \pm 1.61 \\
4.08 \pm 1.29\end{array}$ & 0.22 & n.s. \\
\hline Axial (mm) & $\begin{array}{l}\mathrm{R} \\
\mathrm{L}\end{array}$ & 35 & $\begin{array}{l}6.2 \pm 1.4 \\
6.3 \pm 1.4\end{array}$ & -1.07 & n.s. \\
\hline Sagital (mm) & $\begin{array}{l}\mathrm{R} \\
\mathrm{L}\end{array}$ & 35 & $\begin{array}{l}3.7 \pm 0.8 \\
4.5 \pm 1\end{array}$ & -5.78 & $<0.05$ \\
\hline $\mathrm{CSA}\left(\mathrm{mm}^{2}\right)$ & $\begin{array}{l}\mathrm{R} \\
\mathrm{L}\end{array}$ & 35 & $\begin{array}{l}21.2 \pm 4.6 \\
23.3 \pm 5.8\end{array}$ & -4.10 & $<0.05$ \\
\hline
\end{tabular}

$\mathrm{n}$ - number of volunteers, CSA - Cross sectional area, $\mathrm{R}$ - right, $\mathrm{L}$ - left, n.s - not significant

Table V. Correlation between the grip strength, pinch strength, and ultrasonography measurement results in left-handed volunteers $(\mathrm{n}=35)$

\begin{tabular}{|c|c|c|c|c|c|}
\hline & Axial & Sagital & \multicolumn{2}{|l|}{ CSA } \\
\hline & & $\mathbf{R}$ & $\mathbf{R}$ & & $\mathbf{L}$ \\
\hline \multirow[t]{2}{*}{ Grip (kg) } & $\mathrm{R}$ & $0.64 *$ & $0.46^{*}$ & $0.73^{\prime}$ & \\
\hline & L & $0.52 *$ & $0.48^{*}$ & & $0.67 *$ \\
\hline \multirow[t]{2}{*}{ Pinch-1 (kg) } & $\mathrm{R}$ & $0.43^{*}$ & $0.47 *$ & $0.50^{\prime}$ & \\
\hline & $\mathrm{L}$ & $0.20 \dagger$ & $0.32 \dagger$ & & $0.25 \dagger$ \\
\hline \multirow[t]{2}{*}{ Pinch-2 (kg) } & $\mathrm{R}$ & $0.38 *$ & $0.43^{*}$ & $0.46^{*}$ & \\
\hline & $\mathrm{L}$ & $0.38 *$ & $0.42 *$ & & $0.46^{*}$ \\
\hline \multirow[t]{2}{*}{ Pinch-3 (kg) } & $\mathrm{R}$ & $0.58 *$ & $0.44 *$ & $0.69^{\prime}$ & \\
\hline & $\mathrm{L}$ & $0.47 *$ & $0.42 *$ & & $0.53 *$ \\
\hline \multirow[t]{2}{*}{ Pinch-4 (kg) } & $\mathrm{R}$ & $0.52 *$ & $0.42 *$ & $0.60^{*}$ & \\
\hline & $\mathrm{L}$ & $0.22 \div$ & $0.21 \dagger$ & & $0.31 \dagger$ \\
\hline
\end{tabular}

$*_{r}$ values, $p<0.05$; †r values, $p>0,05$; CSA - cross sectional area; $\mathrm{R}-$ right, $\mathrm{L}$ - left

the differences were significant only in CSA and sagittal thickness US measurements $(\mathrm{p}<0.05)$.

Correlation between US measurements, grip strength and pinch strength results for right-handed and left-handed volunteers are summarized in Table IV and V, respectively. In right-handed volunteers, moderate to strong 
Table VI. Correlations between cross sectional area (CSA) measurements and axial and sagittal thickness measurements in right-handed $(n=54)$ and left-handed volunteers $(n=35)$.

\begin{tabular}{llllll}
\hline & & Axial & \multicolumn{3}{c}{ Sagital } \\
& & $\mathbf{R}$ & $\mathbf{L}$ & $\mathbf{R}$ & $\mathbf{L}$ \\
\hline CSA & right-handed & $0.79^{*}$ & $0.81^{*}$ & $0.76^{*}$ & $0.81^{*}$ \\
& left-handed & $0.87^{*}$ & $0.90^{*}$ & $0.69^{*}$ & $0.69^{*}$ \\
\hline
\end{tabular}

${ }^{*} \mathrm{r}$ values, $\mathrm{p}<0.05, \mathrm{CSA}-$ cross sectional area, $\mathrm{R}$ - right, $\mathrm{L}-$ left

correlations were detected in all measured parameters $(\mathrm{p}<0.05)$. In left handed volunteers, there were moderate to strong statistically significant correlations between all parameters $(p<0.05)$ except for the tip pinch and the three-jaw chuck pinch $(\mathrm{p}>0.05)$. The measurements of CSA were also significantly correlated with axial and sagittal PQ thickness measurements of the corresponding extremity in both right handed and left handed volunteers (Table VI).

\section{Discussions}

The most important finding of this study is that a statistically significant difference exists between dominant and non-dominant hands in CSA and sagittal thickness of the PQ in both right and left handed volunteers. However, US measurements in axial views show a significant difference in favor of the dominant hand only in right-handed volunteers. In addition, although there was no statistically significant differences in the grip strength of the hands in left-handed individuals, dominant side strength measurements were higher in all right-handed volunteers.

Although PQ was described in anatomic texts more than 400 years ago, it did not gain popularity until the biomechanics of the distal radio-ulnar joint became clear [2]. In 1976, Johnson et al [1] reported two distinct heads of PQ. They emphasized the importance of this muscle and postulated that deep head stabilizes the distal radioulnar joint and the superficial head initiates pronation. In addition to these functions, the PQ muscle is the key point indicator in the diagnosis of occult distal forearm fractures on plain radiographs $[4,16]$. However, sensitivity and specificity of the pronator sign has been questioned in the literature regarding the diagnosis of occult distal forearm fractures on plain radiographs $[5,6,8,18]$.

While edematous change of PQ muscle is proposed as an indirect sign for occult distal forearm fracture, loss of bulk of the muscle may reflect anterior inter-osseous nerve palsy. However, isolated PQ atrophy unrelated to anterior inter-osseous nerve entrapment in asymptomatic individuals has been reported in recent studies [10,19]. Tagliafico et al [10] suggested that this might result from the dominance of pronator teres that acts as a primary muscle overriding the PQ activity in the pronation of forearm. In our study, we found that axial and sagittal US measurements for right handed individuals and sagittal US measurements for left handed individuals were significantly higher in dominant hands $(\mathrm{p}<0.05)$. This is also an important finding from the viewpoint that while performing comparative US studies of PQ, hand dominance should be taken into account.

US has distinct advantages in terms of noninvasiveness and cost-effectiveness, and allows for the clear visualization of the PQ in various planes [7,12]. Sonography of PQ can be used in cases of soft tissue swelling of the volar side of wrist, pronation deficit, and loss of handgrip strength in a clinical setting $[7,12]$. By utilization of US images, it is possible to detect occult fractures of distal radius, neoplastic conditions, infectious and granulomatous processes, anterior interosseous syndrome, and rarely for isolated compartment syndrome [7,12]. In our study, we evaluated the sonographic appearance of the PQ both on sagittal and axial images. The sagittal image was used to measure the thickness of radial part of PQ and the axial image was used to measure mainly the thickness of interosseous and ulnar parts [7,12]. These measurements of PQ described in the literature focus mainly on the superficial area because a deep part remains below a line tangential to the cortical bones of the radius and ulna $[7,12]$. However, concerning the ease of the technique, it is more practical to measure the superficial part of the muscle in a clinical setting since a line tangential to two cortical bone surfaces can be clearly drawn. In addition, we also used CSA to evaluate both parts of the PQ with an attempt to include a deep layer to compare with hand dominance and grip strength. Measurements of CSA in US evaluations have been reported in various muscles of the human body in literature but not in PQ previously. Mohseny et al [20] studied the ultrasonographic quantification of the intrinsic hand muscle cross-sectional area and its validity for predicting muscle strength. Thirty-one patients with unilateral ulnar and median nerve injuries were included in their study, and measurements of CSA of intrinsic hand muscles and strength were compared with the contralateral side. They concluded that US is a valid and reliable method to assess the CSA of specific muscles in the hand and this correlates with strength measurements. Katoyose and Magee [21] reported measurement reference standards of CSA of supraspinatus by diagnostic ultrasound in 72 volunteers. They also found that the CSA of the dominant side was significantly larger than the non-dominant side with a high measure of inter-observer reliability. Our technique to measure CSA involves drawing boundaries of PQ muscle area on the axial plane where the maximum thickness of the muscle measured, and the software of US machine 
automatically calculates this CSA. Therefore, we tried to cover both parts of the muscle to compare measurements with the handgrip and side laterality. In our results, CSA measurements were higher in the dominant side and correlated with the dominant site measurements of handgrip strength. The measurements of CSA were also significantly correlated with the axial and sagittal PQ thickness measurements of the corresponding extremity in both right handed and left handed volunteers. These findings related to CSA may be used to search for PQ sonographic swelling in occult distal forearm fractures or for sonographic atrophy of PQ in anterior inter-osseous nerve palsy if validated with further studies in these patient groups.

Grip and pinch strength are commonly employed indices of strength used in hand evaluations. Grips are performed using the whole hand while the pinch involves using the fingers only [14]. Since the deep head of the PQ was active both during pronation and supination, lending support to the theory that it may act primarily as a dynamic distal radio-ulnar joint stabilizer, we also studied grip strength and pinch strength of the hand to utilize the extent of influence concerning stabilizing effect of PQ on wrist [22]. There are a number of studies that consider handgrip strength and pinch forces, mostly tip pinch, lateral pinch and palmar pinch in relation to the upper limb location. Rey et al [23] measured grip strength in pronation and supination, in both the dominant and non-dominant sides in 93 healthy volunteers excluding left handed individuals. Torques were found higher in supination than in pronation and in the dominant side compared to the non-dominant one in their study. They also reported an average ratio (non-dominant/dominant) of 0.85 . In our measurements, the ratio of the non-dominant hand to the dominant hand was higher due to the fact that we performed measurements in the neutral position of forearm. Therefore, it should be kept in mind that measurement of handgrip strength in supination gives more discriminative results.

One of the limitations of our study is that we could not include ambidextrous volunteers since we could not group any person in the handedness survey as mixed handed. Secondly, patients with wrist trauma, fracture and/or operation history were excluded in our study and these directly may alter both strength and US measurements. In addition, CSA measurements of PQ were described for first time in our study, and therefore, this should be validated in further studies including both healthy volunteers and patients.

\section{Conclusion}

Statistically significant difference exists between dominant and non-dominant hands in CSA and sagittal
US measurements of PQ thickness in both right and left handed volunteers, and this is correlated with handgrip strength. Although differences in measurements are considered to be slight, the results are consistent in most of the cases. According to the results of our study, hand dominance should be taken into account while evaluating PQ bilaterally for both sonographic swelling in an occult distal forearm fracture and sonographic atrophy in anterior inter-osseous nerve palsy. In addition, the method described here to measure CSA of PQ may be utilized in various abnormalities of this muscle reported in literature by validating results in further studies comparing the PQ muscle of both healthy volunteers and patients in right and left handed individuals.

\section{Conflict of interest: none}

\section{References}

1. Johnson RK, Shrewsbury MM. The pronator quadratus in motions and in stabilization of the radius and ulna at the distal radioulnar joint. J Hand Surg Am 1976; 1: 205-209.

2. Stuart PR. Pronator quadratus revisited. J Hand Surg Br 1996; 21: 714- 722 .

3. Gordon KD, Pardo RD, Johnson JA, King GJ, Miller TA. Electromyographic activity and strength maximum isometric pronation and supination efforts in healthy adults. J Orthop Res 2004; 22: 208-213.

4. Shin WJ, Kim JP, Kim JS, Park HJ. Sonographic Quantification of Pronator Quadratus Activity During Gripping Effort. J Ultrasound Med 2015; 34: 2269-2278.

5. MacEwan DW. Changes due to trauma in the fat plane overlying the pronator quadratus muscle: a radiologic sign. Radiology 1964; 82: 879-886.

6. Annamalai G, Raby N. Scaphoid and pronator fat stripes are unreliable soft tissue signs in the detection of radiographically occult fractures. Clin Radiol 2003; 58: 798-800.

7. Sato J, Ishii Y, Noguchi H, Takeda M, Toyabe S. Sonographic appearance of the pronator quadratus muscle in healthy volunteers. J Ultrasound Med 2014; 33: 111-117.

8. Sato J, Ishii Y, Noguchi H, Toyabe S. Sonographic swelling of pronator quadratus muscle in patients with occult bone injury. BMC Med Imaging 2015; 15 : 9.

9. Sun B, Zhang D, Gong W, et al. Diagnostic value of the radiographic muscle-to-bone thickness ratio between the pronator quadratus and the distal radius at the same level in undisplaced distal forearm fracture. Eur J Radiol 2016; 85: 452-458.

10. Tagliafico A, Perez MM, Padua L, Klauser A, Zicca A, Martinoli C. Increased reflectivity and loss in bulk of the pronator quadratus muscle does not always indicate anterior interosseous neuropathy on ultrasound. Eur J Radiol 2013; 82: 526-529.

11. Nicholls ME, Thomas NA, Loetscher T, Grimshaw GM. The Flinders Handedness survey (FLANDERS): a brief 
measure of skilled hand preference. Cortex 2013; 49: 2914 2926.

12. Créteur V, Madani A, Brasseur JL. Pronator quadratus imaging. Diagn Interv Imaging 2012; 93: 22-29.

13. Pryce JC. The wrist position between neutral and ulnar deviation that facilitates maximum power grip strength. J Biomech 1980; 13: 505-511.

14. Roman-Liu D. Analysis of maximum force of tip pinch, lateral pinch and palmer pinch in relation to maximum handgrip force. Biol Sport 2003; 20: 303-319.

15. Mathiowetz V, Weber K, Volland G, Kashman N. Reliability and validity of grip and pinch strength evaluation. J Hand Surg Am 1984; 9: 222-226.

16. Schmidt RT, Toews JV. Grip strength as measured by the Jamar dynamometer. Arch Phys Med Rehabil 1970; 51: 321-327.

17. Thorngren KG, Werner CO. Normal grip strength. Acta Orthop Scand 1979; 50: 255-259.

18. Zammit-Maempel I, Bisset RA, Morris J, Forbes WS. The value of soft tissue signs in wrist trauma. Clin Radiol 1988; 39: 664-668.
19. Hinds RM, Gottschalk MB, Capo JT. The pronator quadratus and distal anterior interosseous nerve: a cadaveric study. J Wrist Surg 2015; 4: 183-187.

20. Mohseny B, Nijhuis TH, Hundepool CA, Janssen WG, Selles RW, Coert JH. Ultrasonographic quantification of intrinsic hand muscle cross-sectional area; reliability and validity for predicting muscle strength. Arch Phys Med Rehabil 2015; 96: 845-853.

21. Katayose M, Magee DJ. The cross-sectional area of supraspinatus as measured by diagnostic ultrasound. J Bone Joint Surg Br 2001; 83: 565-568.

22. Lo HY, Cheng HY. Clinical study of the pronator quadratus muscle: anatomical features and feasibility of pronator-sparing surgery. BMC Musculoskelet Disord 2014; 15: 136.

23. Rey PB, Jardin E, Uhring J, Obert L. Is there any correlation between the strength of pronation and supination of the dominant hand and that of the non-dominant one in righthanded healthy adults: preliminary results. Chir Main 2014; 33: 17-22. 